\title{
MMSE-NP-RISIC-Based Channel Equalization for MIMO-SC-FDE Troposcatter Communication Systems
}

\author{
Zedong Xie, Xihong Chen, Xiaopeng Liu, and Yu Zhao \\ Air and Missile Defense College, Air Force Engineering University, Xian 710051, China \\ Correspondence should be addressed to Zedong Xie; 15129054136@163.com
}

Received 22 April 2016; Revised 8 August 2016; Accepted 25 August 2016

Academic Editor: Roman Wendner

Copyright ( 2016 Zedong Xie et al. This is an open access article distributed under the Creative Commons Attribution License, which permits unrestricted use, distribution, and reproduction in any medium, provided the original work is properly cited.

\begin{abstract}
The impact of intersymbol interference (ISI) on single-carrier frequency-domain equalization with multiple input multiple output (MIMO-SC-FDE) troposcatter communication systems is severe. Most of the channel equalization methods fail to solve it completely. In this paper, given the disadvantages of the noise-predictive (NP) MMSE-based and the residual intersymbol interference cancellation (RISIC) equalization in the single input single output (SISO) system, we focus on the combination of both equalization schemes mentioned above. After extending both of them into MIMO system for the first time, we introduce a novel MMSE-NP-RISIC equalization method for MIMO-SC-FDE troposcatter communication systems. Analysis and simulation results validate the performance of the proposed method in time-varying frequency-selective troposcatter channel at an acceptable computational complexity cost.
\end{abstract}

\section{Introduction}

Large capacity troposcatter communication not only plays an important role in the military communications, but also has great potential in other aspects [1]. With the further increases of the required bandwidth and high-speed data transmission, the influence of multipath delay spread of troposcatter channel is becoming more and more prominent [2]. The typical delay spread is extending over tens or hundreds of bit intervals. Furthermore, the significant time-varying Doppler shift mainly due to relative motion of the scatterer causes not only rapid fluctuation in the fading channel response but also compression or dilation of signal waveforms. Thereby, the time-varying frequency-selective fading appears to be more and more severe along with the delay's increase.

In order to combat the multipath fading when the delay spread of channel impulse response (CIR) is large, some scenarios have been devised [3]. The traditional single-carrier time-domain equalization (SC-TDE) typically requires a number of multiplications per symbol that is proportional to the maximum expected channel impulse response length, which results in high computational complexity and rather slow convergence speed [4]. Further increasing of data rates imposes a greater challenge on SC-TDE system. The orthogonal frequency division multiplexing (OFDM) technique [5] has some advantages such as good antifading capability, high spectrum efficiency, and low complexity, but it also has some disadvantages such as high peak-to-average power ratio (PAPR) of its signal and its sensitivity to carrier frequency offset and phase noise [5], so it is not the best applicable one for troposcatter communication whose transmission power is severely limited.

To achieve the goal of favorable trade-off between performance in severe multipath fading channel and complexity of signal processing, the single-carrier frequency-domain equalization (SC-FDE) has recently attracted increased interest because of its similar performance, efficiency, and low signal processing complexity advantages as OFDM, and in addition it is less sensitive than OFDM to radio frequency (RF) impairments such as power amplifier nonlinearities. Consequently, by performing various operations in the frequency domain, through the discrete Fourier transform (DFT), the complexity of processing can be reduced [6].

In SC-FDE systems, linear equalization is simple and practical, but it does not do well in noise and intersymbol 
interference (ISI) suppression [7]. In the literature, the decision feedback equalization (DFE) with a hybrid structure (H-DFE) $[8,9]$, where the feedforward (FF) filter is realized in the frequency domain (FD) while the feedback (FB) filter is realized in the time domain (TD), is derived to cancel the ISI with the disadvantages of relatively high design complexity and error-propagation phenomena. Likewise, there is an equivalent algorithm which is called noise-predictive DFE (NP-DFE) [10]; it is known as suboptimal DFE because the FF filter and the noise predictor (NP) are independently designed, while the FF filter and the FB filter in the conventional H-DFE structure are jointly designed. What is more, residual intersymbol interference cancellation (RISIC) algorithm based minimum mean square error (MMSE) criteria can be utilized to mitigate residual intersymbol interference (RISI), which is also a kind of decision feedback equalization, and it avoids the computation of matrix inversion [10]. As a matter of fact, NP-DFE algorithm only considers the channel noise of the system, while RISIC algorithm takes into account the RISI of the system. Therefore, nonlinear feedback and iteration mechanism are necessarily utilized to improve the performance. Iterative block decision feedback equalization (IBDFE) is an effective nonlinear algorithm with a disadvantage of rather high computational complexity [11].

After recalling the general methods to antagonize serious multipath fading, a novel MMSE-NP-RISIC scheme with advantage of alleviating the effect of both noise and RISI is proposed in this paper. The proposed MMSE-NP-RISIC scheme is the conjunction of the noise-predictive MMSEbased equalization and RISIC algorithms as mentioned previously. Applying the FF filters based on MMSE criterion, which compensates for the frequency-selective fading channel's variations of amplitude and phase, we obtain the elementary time-domain sequence. The following procedure is subdivided into two segments. The noise term is predicted by exploitation of the deterministic characteristic of unique word (UW) and the correlation of the noise at the output for frequency-domain equalization (FDE). And the RISI term can be estimated by the fast Fourier transform (FFT) and the inverse FFT (IFFT) algorithms. Simulation results show that the proposed method can achieve better performance at a modest cost.

The rest of the paper is organized as follows. In Section 2, we give summarization about the multipath effect on the high-capacity troposcatter communication systems and the solutions to combat the resulting ISI. Then, the multiple input multiple output (MIMO) system model and equalization schemes are presented in Section 3. And in Section 4, a novel MMSE-NP-RISIC scheme is proposed. Finally, the extensive simulations are presented in Section 5 and the conclusions are drawn in Section 6.

Notation. Matrices and column vectors are denoted by bold uppercase and lowercase letters, respectively. $E(\cdot)$ denotes the expectation operator. Superscripts $(\cdot)^{T}$ and $(\cdot)^{H}$ stand for the transpose and conjugate transpose operators. $\mathbf{I}_{M}$ denotes the identity matrix of dimension $M . \otimes$ is the Kronecker product.

\section{Multipath Effects on Troposcatter Channel}

We know that the specific type of fading for the corresponding receiver depends on both the transmission scheme and channel characteristics. The transmission scheme is specified with signal parameters such as signal bandwidth and symbol period. Meanwhile, troposcatter channels can be characterized by two different channel parameters, multipath delay spread and Doppler spread, each of which causes time dispersion and frequency dispersion, respectively [12].

Note that any received signal in the propagation environment for a troposcatter channel can be considered as the sum of the received signals from an infinite number of scatters. By the center limit theorem, the received signal can be represented by a Gaussian random variable. The amplitude, phase, and multipath-delayed components from different multipaths are time-variant, which are known as multipath effects.

In terms of time dispersion, a transmit signal may undergo fading over a frequency domain either in a selective or nonselective manner, which is referred to as frequencyselective fading or frequency-nonselective fading, respectively. Due to time dispersion according to multipaths, channel response varies with frequency. As mentioned earlier, transmit signal undergoes frequency-nonselective fading when the troposcatter channel has a constant amplitude and linear phase response only within channel bandwidth narrower than the signal bandwidth [2]. In this case, the channel impulse response has a larger delay spread than a symbol period of the transmit signal. Because of the short symbol duration as compared to the multipath delay spread, multiple-delayed copies of the transmit signal are significantly overlapped with the subsequent symbol, incurring ISI.

For the troposcatter channel, multipath delay spread usually can be described by bilateral multipath delay spread, denoted as $2 \sigma$, which constantly varies from 10 to 500 nanoseconds with the system parameters such as communication distance and antenna aperture. Particularly, in troposcatter communication systems, the empiric formula of $2 \sigma$ applicable to engineering is given as [13]

$$
2 \sigma=\frac{1.2 d^{2}}{f_{r} d_{a} R_{e}},
$$

where $d$ is the communication distance, $f_{r}$ denotes the transmitter frequency, and $d_{a}$ and $R_{e}$ are antenna aperture of the transmitter and the corresponding earth equivalent radius, respectively.

The ratio of bilateral multipath delay spread to symbol period of the transmit signal, denoted as $2 \sigma / T$, is the most effective parameter to measure ISI of troposcatter communication systems, with $T$ denoting the symbol period. In the following and without loss of generality, we can assume that $d=150 \mathrm{~km}, f_{r}=4.7 \mathrm{GHz}, d_{a}=2.4 \mathrm{~m}$, and $R_{e}=8500 \mathrm{~km}$. Here, by substituting them into (1), bilateral multipath delay spread is equal to $2 \sigma=282 \mathrm{~ns}$. Given a troposcatter communication system with the data rate of $8 \mathrm{Mbps}$, we can figure out that $T=192 \mathrm{~ns}, 2 \sigma / T \approx 1.42$. This in turn means that how to mitigate ISI is a question for troposcatter communication systems with high-speed data transmission. 


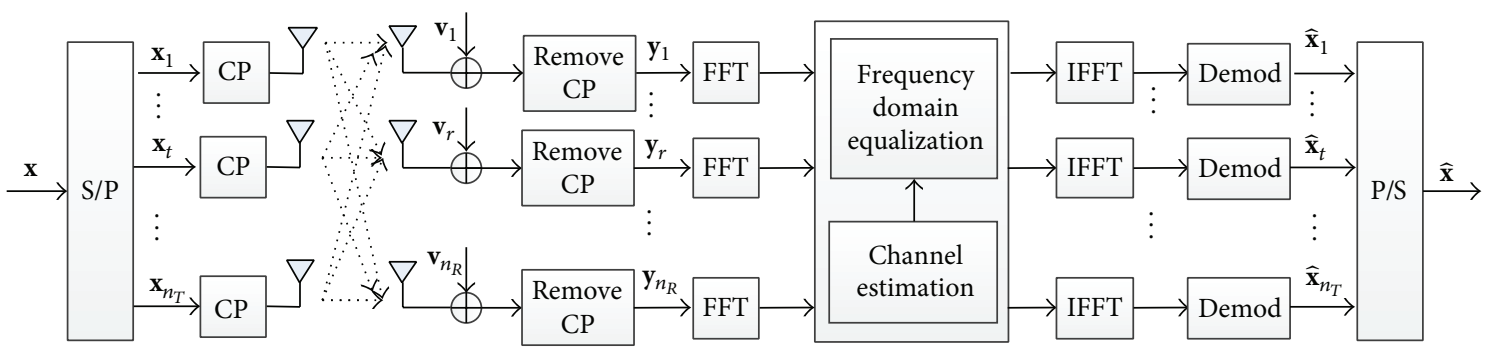

FIGURE 1: MIMO-SC-FDE system architecture with spatial multiplexing.

As long as $2 \sigma / T$ is small enough, the current symbol does not affect the subsequent symbol as much over the next symbol period, implying that ISI is not significant and thereby we can take into account some straightforward measures such as adaptive predistortion technique to combat it. However, when $2 \sigma / T$ is large, some more complex equalization technology must be utilized to mitigate the ISI of the system aroused by multipath effects existing in troposcatter communication. As a matter of fact, under the condition that channel fading is not severe, SCTDE can do well in eliminating ISI. With the increase of transmission rate, the computational complexity of SC-TDE becomes unacceptable. As mentioned above, SC-FDE may be applicable to practical systems over troposcatter multipath channel with favorable trade-off in performance and complexity.

\section{System Model and Equalization Schemes}

3.1. The System Model. We consider a spatial multiple MIMO system. The baseband equivalent system model of the MIMO-SC-FDE transceiver is shown in Figure 1. Let $n_{T}$ and $n_{R}$ be the number of transmit and receive antennas, respectively.

At the transmitter, the data stream is subdivided into $n_{T}$ independent branches by serial-parallel converter. And then they are grouped by the length of $N$. At the beginning of transmit signal block and in the ending of each grouped data stream, a fixed UW sequence known to the receiver is inserted periodically with the length of $N_{c}$. For avoiding interblock interference (IBI), we assume that $N_{c}$ is not less than $L$, the length of CIR.

The data stream at each branch can be denoted as $\mathbf{x}_{t}=$ $\left[x_{t}(1), \ldots, x_{t}(n), \ldots, x_{t}(N)\right]^{T}$, for $t=1, \ldots, n_{T}$. At the receiver, the time-domain MIMO system can be expressed as [14]

$$
\mathbf{y}=\mathbf{h} \mathbf{x}+\mathbf{v}
$$

where the input vector is $\mathbf{x}=\left[\mathbf{x}_{1}^{T}, \mathbf{x}_{2}^{T}, \ldots, \mathbf{x}_{n_{T}}^{T}\right]^{T}$ and the additive noise vector is $\mathbf{v}=\left[\mathbf{v}_{1}^{T}, \mathbf{v}_{2}^{T}, \ldots, \mathbf{v}_{n_{R}}^{T}\right]^{T}$, where $\mathbf{v}_{r}$ for $r=1, \ldots, n_{R}$ denotes white Gaussian noise vector with zero mean and covariance $\sigma_{n}^{2} \mathbf{I}_{n_{R}}$. The output matrix $\mathbf{y}=$ $\left[\mathbf{y}_{1}^{T}, \mathbf{y}_{2}^{T}, \ldots, \mathbf{y}_{n_{R}}^{T}\right]^{T}$ with

$$
\mathbf{y}_{r}=\sum_{t=1}^{n_{T}} \mathbf{h}_{r, t} \mathbf{x}_{t}+\mathbf{v}_{r}
$$

and the time-domain matrix $\mathbf{h}=\left\{\mathbf{h}_{r, t}\right\}$ is an $N n_{R} \times N n_{T}$ matrix whose $(r, t)$ th subchannel impulse response $\mathbf{h}_{r, t}$ can be expressed as an $N \times N$ circulant matrix with the main diagonal value equal to $h_{r, t}(1)$ and the first column equal to $\left[h_{r, t}(1), h_{r, t}(2), \ldots, h_{r, t}(L), 0, \ldots, 0\right]^{T}$, where $h_{r, t}(l)$ for $l=$ $1, \ldots, L$ is the $l$ th tap coefficient of $L$-length channel timedomain impulse response between the $t$ th transmit antenna and the $r$ th receive antenna.

Since $\mathbf{h}_{r, t}$ is a circulant matrix, it has the eigendecomposition [15]:

$$
\mathbf{h}_{r, t}=\mathbf{F}_{N}^{H} \mathbf{H}_{r, t} \mathbf{F}_{N},
$$

where $\mathbf{F}_{N}$ is the normalized DFT matrix of size $N \times N$; that is, its $(p, q)$ th element is given by

$$
\begin{aligned}
{\left[\mathbf{F}_{N}\right]_{p, q}=\frac{1}{\sqrt{N}} e^{-j 2 \pi(p-1)(q-1) / N}, } & \\
& 1 \leq p \leq N, 1 \leq q \leq N .
\end{aligned}
$$

It is easy to prove that $\mathbf{F}_{N}^{H}$ is the corresponding matrix performing IDFT operation. $\mathbf{H}_{r, t}$ is an $N \times N$ diagonal matrix with the main diagonal entries given by the channel frequency responses, which can be expressed as

$$
\left[\mathbf{H}_{r, t}\right]_{p p}=\sum_{l=1}^{L} h_{r, t}(l) \cdot e^{-j 2 \pi(l-1)(p-1) / N}, \quad 1 \leq p \leq N .
$$

Define $\mathbf{D}_{K}=\mathbf{I}_{K} \otimes \mathbf{F}_{N}$. With the property $\mathbf{D}_{n_{T}}^{H} \mathbf{D}_{n_{T}}=\mathbf{I}_{N n_{T}}$, we can obtain the frequency-domain MIMO system model as

$$
\begin{aligned}
\mathbf{Y} & =\mathbf{D}_{n_{R}} \mathbf{y}=\mathbf{D}_{n_{R}} \mathbf{h} \mathbf{x}+\mathbf{D}_{n_{R}} \mathbf{v}=\mathbf{D}_{n_{R}} \mathbf{h} \mathbf{D}_{n_{T}}^{H} \mathbf{D}_{n_{T}} \mathbf{x}+\mathbf{D}_{n_{R}} \mathbf{v} \\
& =\mathbf{H} \mathbf{X}+\mathbf{V},
\end{aligned}
$$

where the channel frequency-domain response matrix $\mathbf{H}$ is defined by

$$
\mathbf{H}=\mathbf{D}_{n_{R}} \mathbf{h} \mathbf{D}_{n_{T}}^{H}=\left[\begin{array}{ccc}
\mathbf{H}_{1,1} & \cdots & \mathbf{H}_{1, n_{T}} \\
\vdots & \ddots & \vdots \\
\mathbf{H}_{n_{R}, 1} & \cdots & \mathbf{H}_{n_{R}, n_{T}}
\end{array}\right] .
$$


However, the overall channel matrix $\mathbf{H}$ is not diagonal, which does not lead to computational savings in the present form. Here, rearrange the input and output vectors by each frequency bin

$$
\begin{aligned}
& \mathbf{X}=\left[\mathbf{X}_{1}^{T}, \ldots, \mathbf{X}_{k}^{T}, \ldots, \mathbf{X}_{N}^{T}\right]^{T}, \\
& \mathbf{Y}=\left[\mathbf{Y}_{1}^{T}, \ldots, \mathbf{Y}_{k}^{T}, \ldots, \mathbf{Y}_{N}^{T}\right]^{T},
\end{aligned}
$$

where $\mathbf{X}_{k}=\left[X_{1}(k), \ldots, X_{t}(k), \ldots, X_{n_{T}}(k)\right]^{T}$ as the rearranged input and $\mathbf{Y}_{k}=\left[Y_{1}(k), \ldots, Y_{r}(k), \ldots, Y_{n_{R}}(k)\right]^{T}$ as the output one. Similarly, the channel matrix for the $k$ th frequency bin is

$$
\mathbf{H}_{k}=\left[\begin{array}{ccc}
H_{1,1}(k) & \cdots & H_{1, n_{T}}(k) \\
\vdots & \ddots & \vdots \\
H_{n_{R}, 1}(k) & \cdots & H_{n_{R}, n_{T}}(k)
\end{array}\right]
$$

where

$$
H_{r, t}(k)=\left[\mathbf{H}_{r, t}\right]_{k k}=\sum_{l=1}^{L} h_{r, t}(l) \cdot e^{-j 2 \pi(l-1)(k-1) / N} .
$$

The channel matrix $\mathbf{H}$ is converted into a block diagonal matrix, that is, $\mathbf{H}=\operatorname{diag}\left[\mathbf{H}_{1}, \ldots, \mathbf{H}_{k}, \ldots, \mathbf{H}_{N}\right]$. The channel model for each frequency bin is then defined as

$$
\mathbf{Y}_{k}=\mathbf{H}_{k} \mathbf{X}_{k}+\mathbf{V}_{k} \text {. }
$$

In the next equalization process, $\mathbf{Y}$ is equalized by some frequency-domain equalization (FDE) schemes, which can be performed separately for each $k$. The equalized signal is converted from the FD back to the TD by the IFFT and the resulting signal is finally detected by the receiver. Thereby, the design of the equalizer is of great importance to detection performance. Next section, some equalization schemes are discussed in detail at the $k$ th frequency tone in order to simplify the derivation and computation.

\subsection{Some Equalization Schemes}

3.2.1. NP-DFE Scheme. We first consider the NP-DFE scheme in MIMO systems without channel coding or interleaving. The coefficients of the feedforward NP-DFE and the feedback NP-DFE are obtained by minimizing the MSE. We prove that in uncoded systems this scheme has exactly the same performance as that of the conventional FD-DFE scheme. The advantages of NP-DFE are also discussed.

We concentrate on the receiver structure in Figure 2, which consists of a feedforward FDE processed in the frequency domain and a group of NPs processed in the time domain [16]. For simplicity, we assume that all NPs have the same order B. Thus, the coefficients of NPs for different data streams can be derived together in a matrix and vector form. by

By multiplying an $n_{T} \times n_{R}$ matrix $\mathbf{W}_{k}$, the output is given

$$
\mathbf{Z}_{k}=\mathbf{W}_{k} \mathbf{Y}_{k}
$$

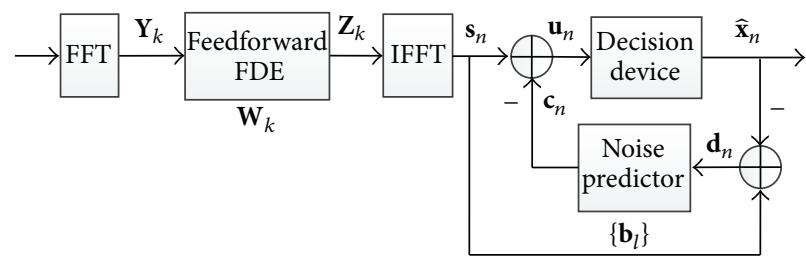

FIgURE 2: The structure of NP-DFE equalizer.

where $\mathbf{W}_{k}$ is the coefficient of the feedforward FDE. By converting $\mathbf{Z}_{k}$ to the time frequency, we will have

$$
\mathbf{s}_{n}=\frac{1}{N} \sum_{k=1}^{N} \mathbf{W}_{k}\left(\mathbf{H}_{k} \mathbf{X}_{k}+\mathbf{V}_{k}\right) \cdot e^{j(2 \pi(k-1) n / N)} .
$$

Then, the data vector before detection can be represented by

$$
\mathbf{u}_{n}=\mathbf{s}_{n}-\mathbf{c}_{n}=\mathbf{s}_{n}-\sum_{l=1}^{B} \mathbf{b}_{l} \cdot \mathbf{d}_{n-l},
$$

where $\mathbf{d}_{n-l}=\mathbf{s}_{n-l}-\widehat{\mathbf{x}}_{n-l}$ and $\mathbf{b}_{l}$ is an $n_{T} \times n_{T}$ matrix representing the coefficients of NPs at the $l$ th tap. Assuming that the feedback symbols are always correct, that is, $\widehat{\mathbf{x}}_{n-l}=$ $\mathbf{x}_{n-l}$, then the error vector is given by

$$
\begin{aligned}
\mathbf{e}_{n} & =\mathbf{u}_{n}-\mathbf{x}_{n}=\mathbf{s}_{n}-\sum_{l=1}^{B} \mathbf{b}_{l} \cdot \mathbf{d}_{n-l}-\mathbf{x}_{n}=\mathbf{d}_{n}-\sum_{l=1}^{B} \mathbf{b}_{l} \cdot \mathbf{d}_{n-l} \\
& =\sum_{l=0}^{B} \mathbf{g}_{l} \cdot \mathbf{d}_{n-l},
\end{aligned}
$$

where

$$
\mathbf{g}_{l}= \begin{cases}\mathbf{I}_{n_{T}} & l=0 \\ -\mathbf{b}_{l} & l=1, \ldots, B\end{cases}
$$

with its average autocorrelation matrix being

$$
E\left\{\mathbf{e}_{n} \mathbf{e}_{n}^{H}\right\}=\sum_{l_{1}=0}^{B} \sum_{l_{2}=0}^{B} \mathbf{g}_{l_{1}} E\left\{\mathbf{d}_{n-l_{1}} \mathbf{d}_{n-l_{2}}^{H}\right\} \mathbf{g}_{l_{2}}^{H} .
$$

The coefficients of the feedforward FDE and the NP can be obtained by minimizing the MSE, which is the trace of (18). Through access to relevant references and noting that $E\left\{\mathbf{X}_{k} \mathbf{X}_{k}^{H}\right\}=\sigma_{x}^{2} \mathbf{I}_{n_{R}}$, we get

$$
\begin{aligned}
\mathbf{W}_{k} & =\sigma_{x}^{2} \mathbf{H}_{k}^{H}\left(\sigma_{x}^{2} \mathbf{H}_{k} \mathbf{H}_{k}^{H}+\sigma_{n}^{2} \mathbf{I}_{n_{R}}\right)^{-1}, \\
\mathbf{b} & =\mathbf{Q}^{-1} \mathbf{q}
\end{aligned}
$$


where

$$
\begin{aligned}
\mathbf{b} & =\left[\begin{array}{c}
\mathbf{b}_{1}^{H} \\
\mathbf{b}_{2}^{H} \\
\vdots \\
\mathbf{b}_{B}^{H}
\end{array}\right], \\
\mathbf{Q} & =\left[\begin{array}{ccccc}
\mathbf{q}_{0} & \mathbf{q}_{1} & \ldots & \mathbf{q}_{B-2} & \mathbf{q}_{B-1} \\
\mathbf{q}_{1}^{H} & \mathbf{q}_{0} & \ldots & \mathbf{q}_{B-3} & \mathbf{q}_{B-2} \\
\vdots & \vdots & \ddots & \vdots & \vdots \\
\mathbf{q}_{B-1}^{H} & \mathbf{q}_{B-2}^{H} & \ldots & \mathbf{q}_{1}^{H} & \mathbf{q}_{0}
\end{array}\right], \\
\mathbf{q} & =\left[\begin{array}{c}
\mathbf{q}_{1}^{H} \\
\mathbf{q}_{2}^{H} \\
\vdots \\
\mathbf{q}_{B}^{H}
\end{array}\right]
\end{aligned}
$$

and where

$$
\mathbf{q}_{l}=\sum_{k=1}^{N}\left(\sigma_{x}^{2} \mathbf{H}_{k} \mathbf{H}_{k}^{H}+\sigma_{n}^{2} \mathbf{I}_{n_{R}}\right)^{-1} e^{j(2 \pi l(k-1) / N)} .
$$

This shows that the proposed NP-DFE is also an optimal design in the sense of MMSE. Furthermore, by taking advantage of the MIMO architecture, different data streams can be reliably detected. The structure of the NP can be dynamically changed without affecting the feedforward FDE.

3.2.2. MMSE-RISIC Scheme. Here, when MMSE criterion is adopted for FDE, the equalized signal can be given by

$$
\mathbf{Z}_{k}=\mathbf{W}_{\mathrm{MMSE}} \mathbf{Y}_{k}=\sigma_{x}^{2} \mathbf{H}_{k}^{H}\left(\sigma_{x}^{2} \mathbf{H}_{k} \mathbf{H}_{k}^{H}+\sigma_{n}^{2} \mathbf{I}_{n_{\mathrm{R}}}\right)^{-1} \mathbf{Y}_{k} .
$$

In fact, the MMSE-based equalization algorithm is tradeoff between the channel noise and the RISI. Given the existence of RISI, FD-DFE and NP-DFE are proposed to alleviate or eliminate residual intersymbol interference. But their performance greatly depends on the order of feedback filter operated in the time domain. Consequently, computational complexity becomes rather high with the increase of the order.

MMSE-RISIC equalization is on the base of MMSE equalization, which is a new kind of decision feedback equalizer. However, this equalization can only be used in SISO system. Here, we propose novel MMSE-RISIC equalization for MIMO system for the first time.

Figure 3 shows the structure of MMSE-RISIC equalization, in which the RISI of the system is estimated and eliminated in the frequency domain. In order to estimate the RISI of the MIMO system, some manipulations are carried out after the feedforward MMSE-FDE. Specifically, we give the simple mathematical derivations.

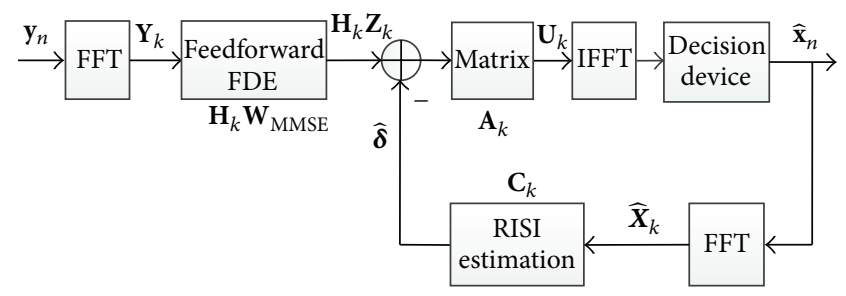

FIGURE 3: The structure of MMSE-RISIC equalizer.

As mentioned previously, (22) can also be rewritten as follows:

$$
\begin{aligned}
\mathbf{Z}_{k}= & \sigma_{x}^{2} \mathbf{H}_{k}^{H}\left(\sigma_{x}^{2} \mathbf{H}_{k} \mathbf{H}_{k}^{H}+\sigma_{n}^{2} \mathbf{I}_{n_{R}}\right)^{-1} \mathbf{Y}_{k} \\
= & \mathbf{H}_{k}^{H}\left(\mathbf{H}_{k} \mathbf{H}_{k}^{H}+\rho \mathbf{I}_{n_{R}}\right)^{-1} \mathbf{H}_{k} \mathbf{X}_{k}+\widetilde{\mathbf{V}}_{k} \\
= & \mathbf{H}_{k}^{H}\left(\mathbf{H}_{k} \mathbf{H}_{k}^{H}\right)^{-1}\left[\mathbf{I}_{n_{R}}-\rho\left(\mathbf{H}_{k} \mathbf{H}_{k}^{H}+\rho \mathbf{I}_{n_{R}}\right)^{-1}\right] \mathbf{H}_{k} \mathbf{X}_{k} \\
& +\widetilde{\mathbf{V}}_{k},
\end{aligned}
$$

where

$$
\begin{aligned}
\rho & =\frac{\sigma_{n}^{2}}{\sigma_{x}^{2}}, \\
\widetilde{\mathbf{V}}_{k} & =\mathbf{H}_{k}^{H}\left(\mathbf{H}_{k} \mathbf{H}_{k}^{H}+\rho \mathbf{I}_{n_{R}}\right)^{-1} \mathbf{V}_{k} .
\end{aligned}
$$

The observation of (23) indicates that we cannot separate out $\mathbf{X}_{k}$ from $\mathbf{Z}_{k}$ just in the method used in SISO system. Here, we consider that by multiplying $\mathbf{H}_{k}$ the relationship between $\mathbf{X}_{k}$ and RISI $\widehat{\boldsymbol{\delta}}$ can become increasingly clear. That is,

$$
\begin{aligned}
\mathbf{H}_{k} \mathbf{Z}_{k} & =\left[\mathbf{I}_{n_{R}}-\rho\left(\mathbf{H}_{k} \mathbf{H}_{k}^{H}+\rho \mathbf{I}_{n_{R}}\right)^{-1}\right] \mathbf{H}_{k} \mathbf{X}_{k}+\widehat{\mathbf{V}}_{k} \\
& =\mathbf{H}_{k} \mathbf{X}_{k}-\rho\left(\mathbf{H}_{k} \mathbf{H}_{k}^{H}+\rho \mathbf{I}_{n_{R}}\right)^{-1} \mathbf{H}_{k} \mathbf{X}_{k}+\widehat{\mathbf{V}}_{k} \\
& =\mathbf{H}_{k} \mathbf{X}_{k}+\widehat{\boldsymbol{\delta}}+\widehat{\mathbf{V}}_{k},
\end{aligned}
$$

where

$$
\begin{aligned}
\widehat{\mathbf{V}}_{k} & =\mathbf{H}_{k} \widetilde{\mathbf{V}}_{k}, \\
\widehat{\boldsymbol{\delta}} & =-\rho\left(\mathbf{H}_{k} \mathbf{H}_{k}^{H}+\rho \mathbf{I}_{n_{R}}\right)^{-1} \mathbf{H}_{k} \mathbf{X}_{k} .
\end{aligned}
$$

Then, the data vector before detection can be represented by

$$
\begin{aligned}
\mathbf{U}_{k} & =\mathbf{H}_{k}^{H}\left(\mathbf{H}_{k} \mathbf{H}_{k}^{H}\right)^{-1}\left(\mathbf{H}_{k} \mathbf{Z}_{k}-\widehat{\boldsymbol{\delta}}\right) \\
& =\mathbf{A}_{k}\left(\mathbf{H}_{k} \mathbf{Z}_{k}-\mathbf{C}_{k} \mathbf{X}_{k}\right),
\end{aligned}
$$

where

$$
\begin{aligned}
\mathbf{A}_{k} & =\mathbf{H}_{k}^{H}\left(\mathbf{H}_{k} \mathbf{H}_{k}^{H}\right)^{-1}, \\
\mathbf{C}_{k} & =-\rho\left(\mathbf{H}_{k} \mathbf{H}_{k}^{H}+\rho \mathbf{I}_{n_{\mathrm{R}}}\right)^{-1} \mathbf{H}_{k} .
\end{aligned}
$$

The RISI estimate value $\widehat{\boldsymbol{\delta}}$ can be greatly used to combat residual intersymbol interference of the MIMO system. In addition, the complexity is low because it mainly needs once FFT and IFFT operations. 


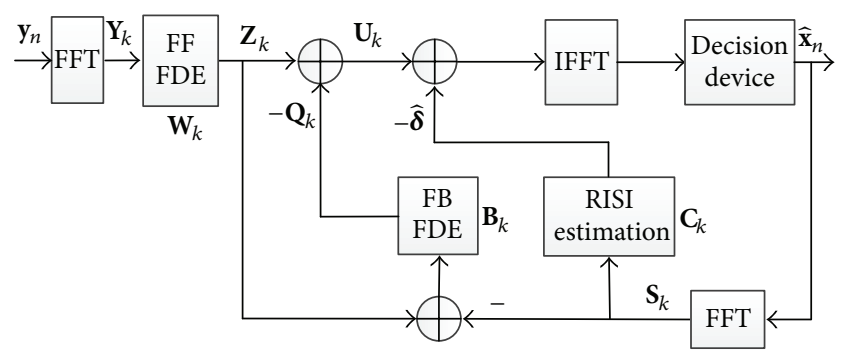

FIgURE 4: The structure of MMSE-NP-RISIC equalization.

\section{The Proposed MMSE-NP-RISIC Scheme}

The algorithm of the NP-DFE scheme only takes into account channel noise. Likewise, the MMSE-RISIC equalization mainly focuses on elimination of the residual intersymbol interference of the MIMO system. Based on what is mentioned above, a novel MMSE-NP-RISIC equalization scheme is proposed.

Figure 4 shows the structure of the proposed MMSENP-RISIC equalization. The NP part and MMSE-RISIC part are both based on MMSE criterion and operated in the frequency domain. Therefore, the NP part is different from the NP-DFE scheme mentioned above, where the feedback filter is operated in the time domain. We analyze and derive the frequency-domain coefficients of both the feedforward FDE and feedback FDE in the MMSE sense. As is shown in Figure 4 , the output vector of the NP part, $\mathbf{U}_{k}$, can be obtained as

$$
\mathbf{U}_{k}=\mathbf{Z}_{k}-\mathbf{Q}_{k}=\mathbf{Z}_{k}-\mathbf{B}_{k}\left(\mathbf{Z}_{k}-\mathbf{S}_{k}\right) \text {. }
$$

Suppose $\mathbf{S}_{k}=\mathbf{X}_{k}$; then the detection error of the NP part in the frequency domain will be given by

$$
\begin{aligned}
\mathbf{E}_{k}= & \mathbf{U}_{k}-\mathbf{X}_{k}=\mathbf{Z}_{k}-\mathbf{B}_{k}\left(\mathbf{Z}_{k}-\mathbf{X}_{k}\right)-\mathbf{X}_{k} \\
= & \left(\mathbf{I}_{n_{R}}-\mathbf{B}_{k}\right) \mathbf{Z}_{k}-\left(\mathbf{I}_{n_{R}}-\mathbf{B}_{k}\right) \mathbf{X}_{k} \\
= & \left(\mathbf{I}_{n_{R}}-\mathbf{B}_{k}\right)\left(\mathbf{W}_{k} \mathbf{H}_{k} \mathbf{X}_{k}+\mathbf{W}_{k} \mathbf{V}_{k}\right)-\left(\mathbf{I}_{n_{R}}-\mathbf{B}_{k}\right) \mathbf{X}_{k} \\
= & \left(\mathbf{I}_{n_{R}}-\mathbf{B}_{k}\right)\left(\mathbf{W}_{k} \mathbf{H}_{k}-\mathbf{I}_{n_{R}}\right) \mathbf{X}_{k} \\
& +\left(\mathbf{I}_{n_{R}}-\mathbf{B}_{k}\right) \mathbf{W}_{k} \mathbf{V}_{k} .
\end{aligned}
$$
be

The autocorrelation matrix of the detection error $\mathbf{e}_{n}$ will

$$
E\left\{\mathbf{e}_{n} \mathbf{e}_{n}^{H}\right\}=\frac{1}{N} E\left\{\sum_{k=1}^{N} \mathbf{E}_{k} \mathbf{E}_{k}^{H}\right\} .
$$

The MSE is the trace of (31). By substituting (30) into (31), differentiating the trace with respect to $\mathbf{W}_{k}$, and setting the result to zero, we get

$$
\mathbf{W}_{k}=\sigma_{x}^{2} \mathbf{H}_{k}^{H}\left(\sigma_{x}^{2} \mathbf{H}_{k} \mathbf{H}_{k}^{H}+\sigma_{n}^{2} \mathbf{I}_{n_{R}}\right)^{-1},
$$

where $\mathbf{W}_{k}$ is the frequency-domain coefficients of the feedforward FDE.
By introducing the constraint that

$$
\sum_{k=1}^{N} \mathbf{B}_{k}=\mathbf{0},
$$

and taking the similar manipulation, we can obtain

$$
\begin{aligned}
\mathbf{B}_{k}=\mathbf{I}_{n_{R}} & -N\left(\sigma_{x}^{2} \mathbf{H}_{k} \mathbf{H}_{k}^{H}+\sigma_{n}^{2} \mathbf{I}_{n_{R}}\right) \\
\cdot & {\left[\sum_{m=1}^{N}\left(\sigma_{x}^{2} \mathbf{H}_{m} \mathbf{H}_{m}^{H}+\sigma_{n}^{2} \mathbf{I}_{n_{R}}\right)\right]^{-1}, }
\end{aligned}
$$

where $\mathbf{B}_{k}$ is the frequency-domain coefficients of the feedback FDE.

Here, we note that the NP part is different from the traditional NP-DFE scheme. In the MMSE-NP-RISIC scheme, FF FDE and FB FDE are both operated in the frequency domain.

Then the MMSE-RISIC part is utilized to further eliminate the residual ISI existing in the output vector of the NP part. Here, we refer to the MMSE-RISIC scheme proposed above and the coefficient matrix can be obtained in a similar approach.

Actually, the input vector of the MMSE-RISIC part, $\mathbf{U}_{k}$, can be rewritten as

$$
\begin{aligned}
\mathbf{U}_{k}= & \mathbf{Z}_{k}-\mathbf{B}_{k}\left(\mathbf{Z}_{k}-\mathbf{S}_{k}\right) \\
= & \mathbf{X}_{k}+\left(\mathbf{I}_{n_{R}}-\mathbf{B}_{k}\right)\left(\mathbf{W}_{k} \mathbf{H}_{k}-\mathbf{I}_{n_{R}}\right) \mathbf{X}_{k} \\
& +\left(\mathbf{I}_{n_{R}}-\mathbf{B}_{k}\right) \mathbf{W}_{k} \mathbf{V}_{k}=\mathbf{X}_{k}+\widehat{\boldsymbol{\delta}}+\widehat{\mathbf{V}}_{k}
\end{aligned}
$$

where

$$
\begin{aligned}
\widehat{\mathbf{V}}_{k} & =\left(\mathbf{I}_{n_{R}}-\mathbf{B}_{k}\right) \mathbf{W}_{k} \mathbf{V}_{k}, \\
\widehat{\boldsymbol{\delta}} & =\left(\mathbf{I}_{n_{R}}-\mathbf{B}_{k}\right)\left(\mathbf{W}_{k} \mathbf{H}_{k}-\mathbf{I}_{n_{R}}\right) \mathbf{X}_{k} .
\end{aligned}
$$

Then, $\mathbf{C}_{k}$, the frequency-domain coefficients of the RISI estimation, can be given by

$$
\mathbf{C}_{k}=\left(\mathbf{I}_{n_{R}}-\mathbf{B}_{k}\right)\left(\mathbf{W}_{k} \mathbf{H}_{k}-\mathbf{I}_{n_{R}}\right),
$$

when calculating $\mathbf{C}_{k}, \mathbf{W}_{k}$ and $\mathbf{B}_{k}$ should be obtained before according to (32) and (34), respectively.

The novel MMSE-NP-RISIC equalization scheme can eliminate the influence of both the channel noise and the residual ISI at the same time, which is the advantage that the existing other methods do not have. It is of great importance especially for the high-capacity MIMO-SC-FDE troposcatter communication systems.

\section{Simulation Analysis}

In this section, we evaluate the performance of the proposed method in terms of the normalized mean squared error (NMSE) and bit error rate (BER) and compare the computational load with other equalization methods.

In the simulated MIMO-SC-FDE system, let the number of transmit and receive antennas be $M=N=2$. A standard 
TABLE 1: The parameters of a 300-kilometer-long troposcatter communications link in North China.

\begin{tabular}{lcccc}
\hline Tap number & Relative time delay (us) & Path gain & Doppler spread (Hz) & Doppler spectrum \\
\hline 1 & 0 & 0.2772 & 100 & Gaussian \\
2 & 0.1 & 0.4130 & 120 & Gaussian \\
3 & 0.2 & 0.7177 & 110 & Gaussian \\
4 & 0.3 & 0.8518 & 100 & Gaussian \\
5 & 0.4 & 0.8184 & 80 & Gaussian \\
6 & 0.5 & 0.6713 & 90 & Gaussian \\
7 & 0.6 & 0.4813 & 85 & Gaussian \\
8 & 0.7 & 0.3055 & 105 & Gaussian \\
9 & 0.8 & 0.1730 & 86 & Gaussian \\
\hline
\end{tabular}

convolutional code with code rate $1 / 2$, constraint length 5 , and octal generator polynomials $(23,35)$ is applied. The coded bits are mapped to QPSK for data (BPSK for UW). The block size has been set to $K=512$, while the UW extension size has been set to $Q=24$ and the data size is $P=K-Q=488$.

By reference to a large amount of measured data, we can choose a typical 300-kilometer-long troposcatter communications link in North China, which is subject to frequencyselective fast fading. Table 1 presents its channel parameters $[17,18]$, in which nine different multiple signal paths are characterized by their relative delay and average power. The length of channel impulse response is $L=9$. We assumed that each channel has a fixed implied response for each block period and that the receiver has perfect synchronization and channel state information. Nevertheless, in the process of the equalization and channel decode, iterative algorithm can only provide a relatively small improvement in the accuracy, but the complexity of it will inevitably increase in multiples. Iterations are not used to obtain the results in simulations.

Here, for convenience of discussion, the implementation method based on the traditional MMSE linear equalization is called MMSE-FDE. And the NP-DFE scheme is noisepredictive decision-feedback detection proposed in [10]. The MMSE-RISIC denotes residual ISI cancellation based minimum mean square error criterion proposed in [11]. To illustrate the degradation due to error propagation phenomenon, the performance of MMSE-NP-RISIC and NP-DFE with corrected symbols fed back is provided. Furthermore, the performance of the matched filter bound (MFB) is also provided as a useful metric to compare equalizer structures. Here, Figures 5 and 6 show the average BER and NMSE performance of the equalization methods in the troposcatter channel mentioned above, respectively.

The ZF-FDE cancels the interference completely without regard to noise amplification. Thereby, the MMSE-FDE improves on this strategy by finding the optimal balance between interference cancellation and noise reduction that minimizes the total MSE. The NP-DFE consists of a linear detector and a linear prediction mechanism that reduces noise variance. The noise-predictive implementation makes it easy to upgrade an existing linear detector by appending relatively simple additional processing. The MMSE-RISIC takes into consideration the fact that there still exists the residual ISI due to imperfect channel equalization, which will

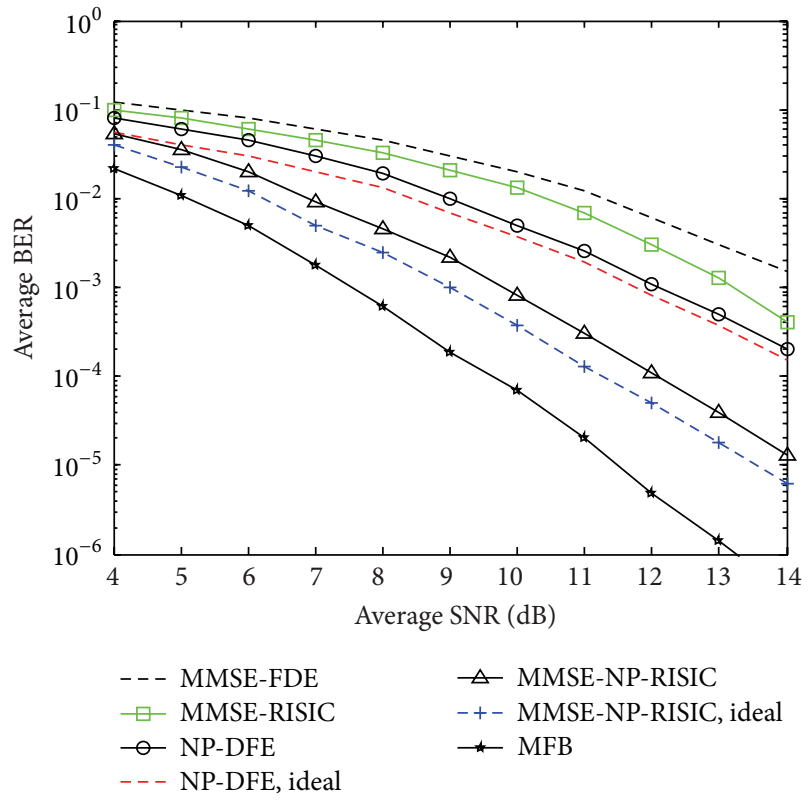

FIGURE 5: Average BER versus average SNR of various equalization schemes in the time-varying frequency-selective troposcatter channel.

severely degrade the system performance. With the initial estimates of MMSE equalizer, the RISIC algorithm is adopted to alleviate the residual ISI. The proposed MMSE-NP-RISIC scheme takes into account the noise term and RISI term of the signal equalized by MMSE-based equalizer, which can further improve the equalizer performance.

For the ideal NP-DFE and ideal MMSE-NP-RISIC, perfect decisions, that is, error-free decisions, are fed back to the FBF. However, in the presence of noise and residual ISI, decision errors are inevitable. The first noise and residual ISI induced error is known as a primary error. As the decision error is fed back through the FBF, instead of cancelling the post-cursor ISI components, it also adds additional interference.

As shown in Figure 5, the proposed MMSE-NP-RISIC method outperforms the other schemes mentioned above. For a BER of $10^{-4}$, the performance degradation of the proposed structure is about $1 \mathrm{~dB}$ when compared to the ideal 


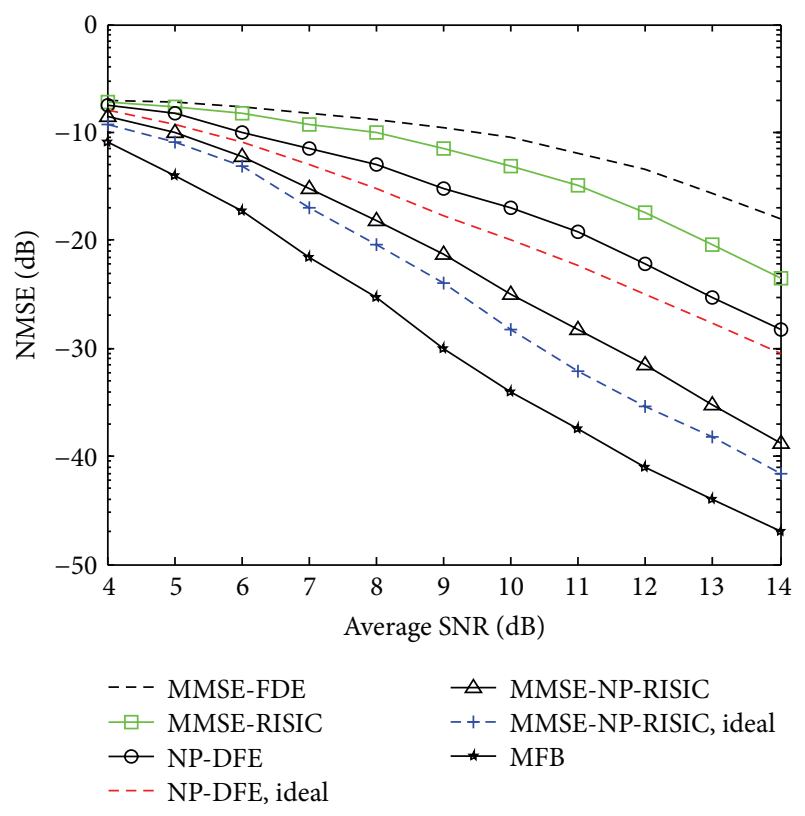

FIGURE 6: NMSE versus average SNR of various equalization schemes in the time-varying frequency-selective troposcatter channel.

MMSE-NP-RISIC. Compared with the NP-DFE and MMSERISIC, the new proposed structure yields SNR gain of about $2 \mathrm{~dB}$ and $3.5 \mathrm{~dB}$ at $\mathrm{BER}=10^{-3}$, respectively. It can be explained as the consequence of two factors: the improved noise predictive part and the RISIC process. In fact, in the proposed structure, the noise predictive part can gradually increase the reliability of the detected data, thus reducing the effects of the error propagation that limits the traditional DFE performance, especially at low SNRs. Moreover, the RISIC process is able to remove the residual ISI existing in the equalized signal. What is more, under the condition SNR = $10 \mathrm{~dB}$, the MMSE-NP-RISIC can decrease the average BER by almost one order of magnitude compared with the NPDFE. In the simulations, we also find that the gaps in BER performance will grow over the increase of the average SNR, which illustrates that the advantage of the proposed scheme is more obvious at relative high SNRs.

However, the proposed MMSE-NP-RISIC still has a gap from the MFB. The performance degradation of the DFE with respect to the MFB can be decomposed into two parts. The first is the error propagation gap. The second component is known as the gap from the MFB. Note that the gap from the MFB increases with increasing SNR. This is due to the fact that at high SNR scenarios ISI is the dominant factor. We can also come to the conclusion that the MMSE-RISIC and NPDFE can only slightly improve the BER performance of the system, while the MMSE-NP-RISIC will bring a significant increase.

In addition, Figure 6 illustrates NMSE performance of the proposed scheme. When average SNR $<6 \mathrm{~dB}$, MMSE-NPRISIC exhibits similar performance with MMSE-RISIC and NP-DFE, although their related implementation costs differ significantly, whereas, under the condition that $\mathrm{SNR}>8 \mathrm{~dB}$,
TABLE 2: The CMUL per output sample needed for signal processing.

\begin{tabular}{lccc}
\hline Structure & \multicolumn{2}{c}{$\begin{array}{c}\text { Computational complexity of the system } \\
\text { Number of complex } \\
\text { multiplications }\end{array}$} & $\begin{array}{c}\text { Simulation scenario } \\
\text { MMSE-RISIC }\end{array}$ \\
NP-DFE & $\left(K \log _{2} K+K\right) / P$ & 11 & 20 \\
MMSE-NP-RISIC & $\left(2 K \log _{2} K+K+B K\right) / P$ & $B=9$ & 22 \\
\hline
\end{tabular}

TABLE 3: The CMUL for the equalizer design.

\begin{tabular}{|c|c|c|}
\hline \multirow{2}{*}{ Structure } & \multicolumn{2}{|c|}{$\begin{array}{l}\text { Computational complexity of the equalizer } \\
\text { design }\end{array}$} \\
\hline & $\begin{array}{c}\text { Number of complex } \\
\text { multiplications }\end{array}$ & Simulation scenario \\
\hline MMSE-RISIC & $2 K$ & 1024 \\
\hline NP-DFE & $B^{2}+(B+1) K$ & $B=9$ \\
\hline MMSE-NP-RISIC & $5 M K+3 M Q$ & 5264 \\
\hline
\end{tabular}

the proposed method has a smaller NMSE than the others, especially at relative high SNRs. What is more, error propagation results in an increased probability of error during the subsequent symbol decision. Hence, the NMSE performance degradation of the proposed structure is severe when compared to the ideal MMSE-NP-RISIC.

The computational complexity of the various schemes is mainly evaluated in terms of the number of complex multiplications (CMULs), for both the signal processing and filter design. Table 2 presents the CMUL per output sample needed for signal processing, and Table 3 shows the CMUL for the equalizer design [8]. As shown in the tables, the overall computational load of the proposed MMSE-NPRISIC is higher than the other methods mentioned above. The improvements of the performance are at an increased complexity cost. From the theoretical analysis and the time complexity collected during the run, the increase of the proposed scheme is acceptable. The frequency-domain equalization can be performed for each frequency bin to further reduce the computational load.

\section{Conclusion}

In this paper, we have mainly presented a novel channel equalization scheme for MIMO-SC-FDE troposcatter communication systems. Here, we firstly analyze the wellknown methods to combat the ISI existing in large capacity troposcatter communication. Then, we focus on the MIMOSC-FDE system model and some equalization schemes. Based on the MMSE criterion, the NP-DFE equalization and MMSE-RISIC equalization schemes are proposed to make it applicable to MIMO system for the first time, respectively. Furthermore, we present a new MMSE-NPRISIC channel equalization scheme to combat the ISI of the troposcatter channel. The frequency-domain channel estimation and equalization can be performed separately for 
each frequency bin to further reduce the computational complexity of the proposed method. Numerical results show that compared with the existing methods mentioned above the proposed equalization scheme achieves better performance at an acceptable computational load cost in MIMO-SC-FDE troposcatter communication systems.

\section{Competing Interests}

The authors declare that there is no conflict of interests regarding the publication of this paper.

\section{Acknowledgments}

This work was supported by National Natural Science Fund of China under Grant no. 60971100.

\section{References}

[1] F. A. Gunther, "Tropospheric scatter communications past, present, and future," IEEE Spectrum, vol. 3, no. 9, pp. 79-100, 1966.

[2] M. Zhang, Tropospheric Scatter Propagation, Publishing House of Electronics Industry, Beijing, China, 2004.

[3] J. G. Proakis and S. Masoud, Digital Communications, McGrawHill Companies, New York, NY, USA, 5th edition, 2009.

[4] Y. Zhu and K. B. Letaief, "Single carrier frequency domain equalization with noise prediction for broadband wireless systems," in Proceedings of the Global Telecommunications Conference (GLOBECOM '04), vol. 5, pp. 3098-3102, IEEE, Dallas, Tex, USA, November-December 2004.

[5] L. Hanzo, M. Münster, and B. J. Choi, OFDM and MCCDMA for Broadband Multi-User Communications, WLANs and Broadcasting, Chichester, UK, 2003.

[6] F. Pancaldi, G. M. Vitetta, R. Kalbasi, N. Al-Dhahir, M. Uysal, and H. Mheidat, "Single-carrier frequency domain equalization," IEEE Signal Processing Magazine, vol. 25, no. 5, pp. 37-56, 2008.

[7] C. Zhang, Z. Wang, C. Pan, S. Chen, and L. Hanzo, "Lowcomplexity iterative frequency domain decision feedback equalization," IEEE Transactions on Vehicular Technology, vol. 60, no. 3, pp. 1295-1301, 2011.

[8] N. Benvenuto and S. Tomasin, "On the comparison between OFDM and single carrier modulation with a DFE using a frequency-domain feedforward filter" IEEE Transactions on Communications, vol. 50, no. 6, pp. 947-955, 2002.

[9] D. Falconer, S. L. Ariyavisitakul, A. Benyamin-Seeyar, and B. Eidson, "Frequency domain equalization for single-carrier broadband wireless systems," IEEE Communications Magazine, vol. 40, no. 4, pp. 58-66, 2002.

[10] Y. Zhu and K. B. Letaief, "Single carrier frequency domain equalization with time domain noise prediction for wideband wireless communications," IEEE Transactions on Wireless Communications, vol. 5, no. 12, pp. 3548-3557, 2006.

[11] Y. Zhi and L. Ze-Ming, "A novel decision feedback equalizer for SC-FDE system," Semiconductor Technology, vol. 32, no. 9, pp. 800-803, 2007.

[12] Y. S. Cho, J. Kim, and W. Y. Yang, MIMO-OFDM Wireless Communications with MATLAB, John Wiley \& Sons, Singapore, 2010.
[13] R. Li, X. Ren, and Y. Liu, "Summarization of new developments of digital troposcatter transmission technology," Radio Communications Technology, vol. 33, no. 3, pp. 8-13, 2007.

[14] B. Ng, C.-T. Lam, and D. Falconer, "Turbo frequency domain equalization for single-carrier broadband wireless systems," IEEE Transactions on Wireless Communications, vol. 6, no. 2, pp. 759-767, 2007.

[15] A. Stamoulis, S. N. Diggavi, and N. Al-Dhahir, "Intercarrier interference in MIMO OFDM," IEEE Transactions on Signal Processing, vol. 50, no. 10, pp. 2451-2464, 2002.

[16] X. Yuan, Q. Guo, X. Wang, and L. Ping, "Evolution analysis of low-cost iterative equalization in coded linear systems with cyclic prefixes," IEEE Journal on Selected Areas in Communications, vol. 26, no. 2, pp. 301-310, 2008.

[17] M. Hu, X. Chen, and B. Liu, "An improved singular-valueddecomposition channel estimation algorithm in troposcatter communications," Telecommunication Engineering, vol. 49, no. 8, pp. 69-72, 2009.

[18] X. Chen, Study on Diversity Combining of High Rate Troposcatter Communication, Nanjing University of Science and Technology, Nanjing, China, 2007. 


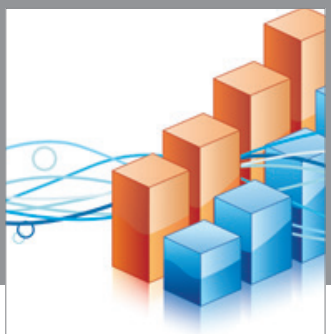

Advances in

Operations Research

vatem alat4

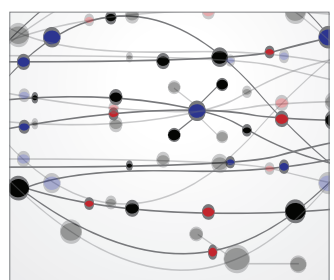

\section{The Scientific} World Journal
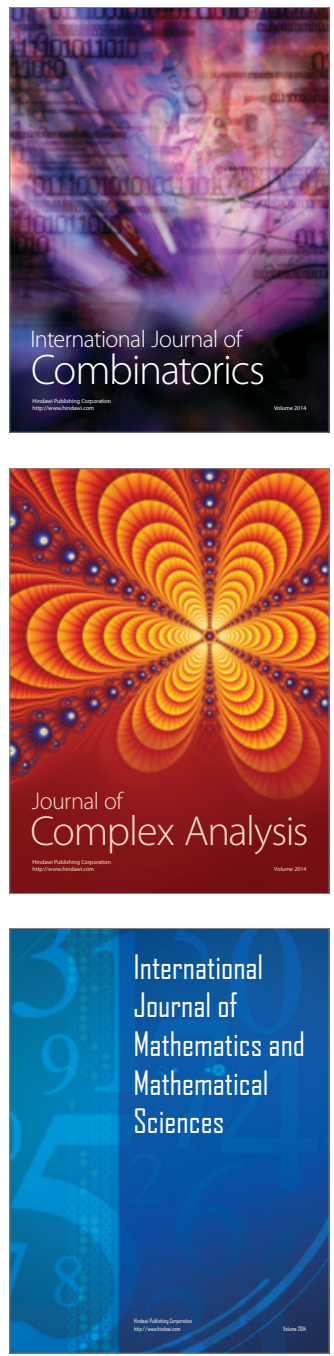
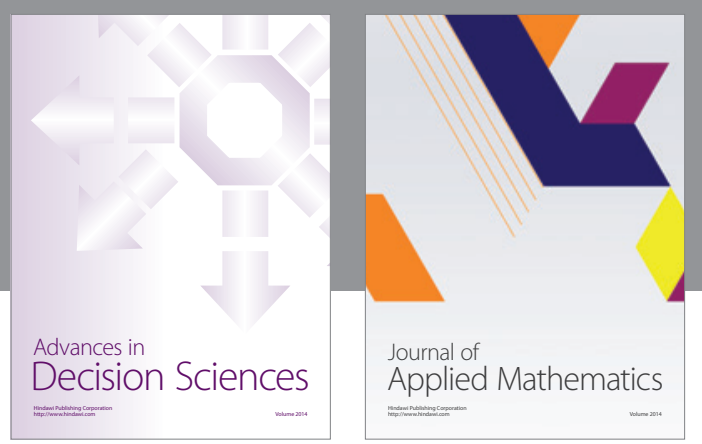

Algebra

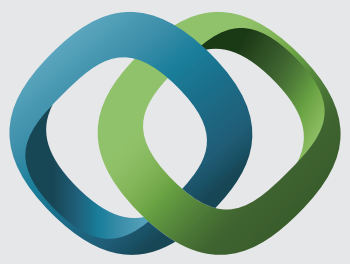

\section{Hindawi}

Submit your manuscripts at

http://www.hindawi.com
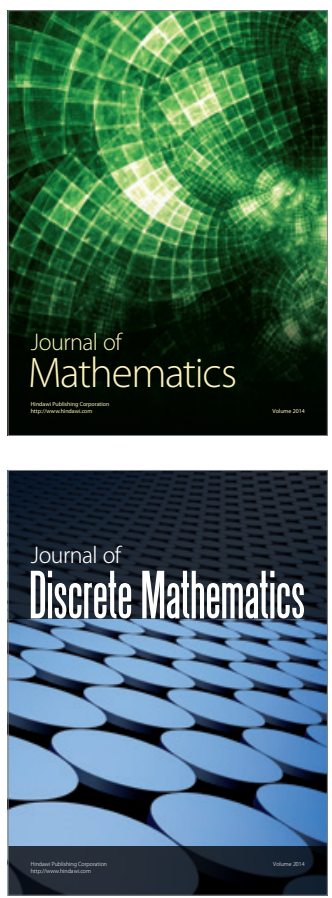

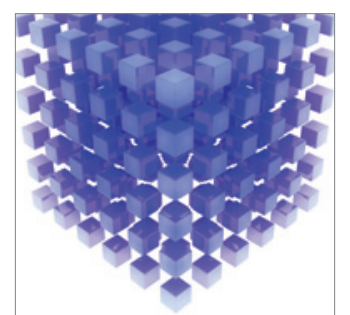

Mathematical Problems in Engineering
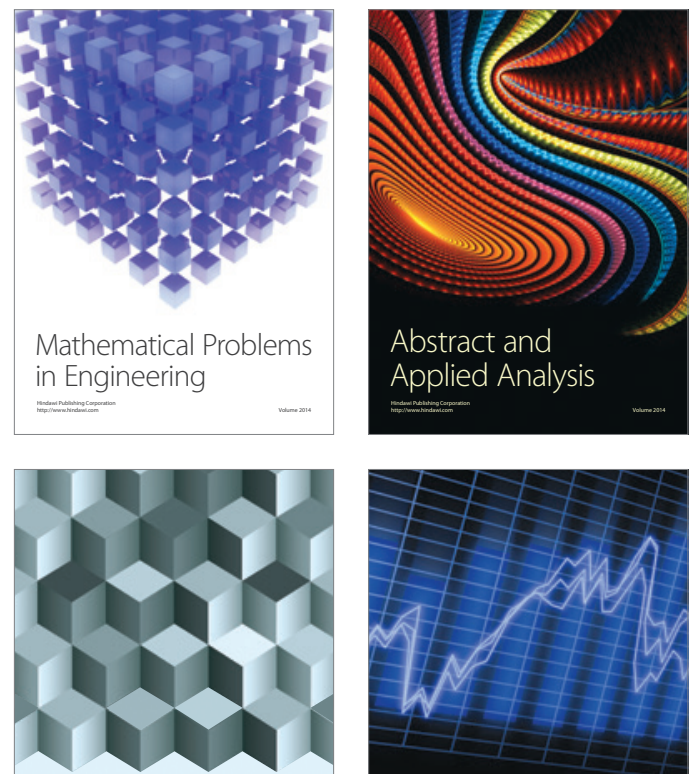

Journal of

Function Spaces

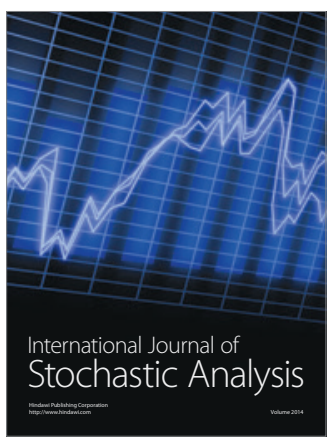

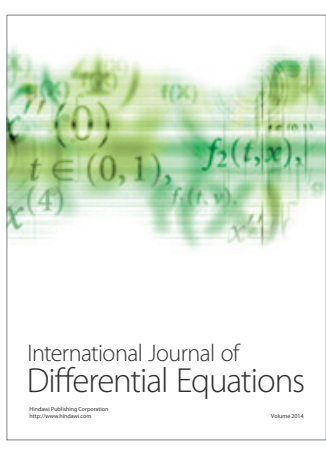
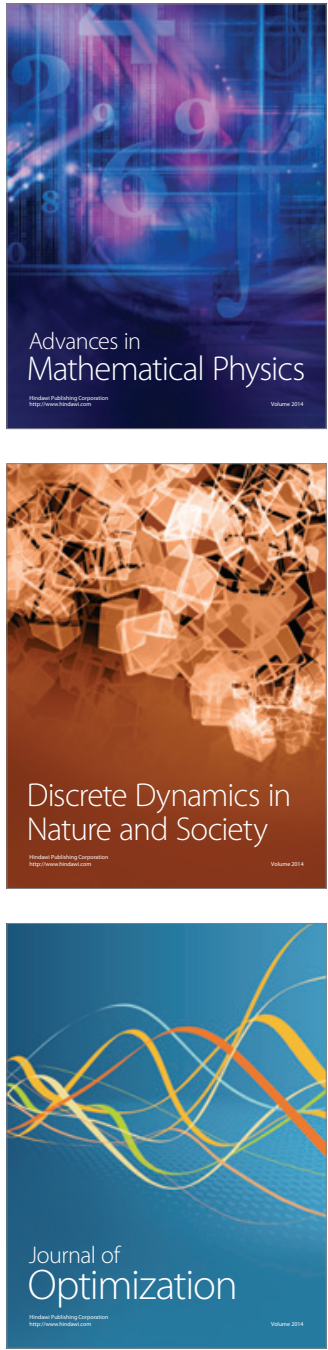